\title{
ON QUERMASSINTEGRAL AND WILLS CONJECTURE
}

\section{FANGWEI CHEN}

Abstract. In this paper we investigate the relative quermassintegrals of convex bodies. We obtain an lower bound of the relative quermassintegral $W_{i}(K ; E)$. Specially, we give another stronger Wills inequality.

Mathematics subject classification (2010): Primary 52A40, 53A20; Secondary 52A10. Keywords and phrases: Bonessen inequality, quermassintegral, Wills conjecture.

\section{REFERENCES}

[1] J. BoKOWSKI, Eine verschärfte ungleichung zwischen Volumen, Oberfläche und Inkuglradius im $R^{n}$, Elem. Math., 28 (1973), 43-44.

[2] T. Bonnesen, Les problèmes des Isopérimètres et des Isépiphanes, Gauthier-Villars, Paris, 1929.

[3] N. S. Brannen, The Wills conjecture, Trans. Amer. Math. Soc., 349 (1997), 3977-3987.

[4] V. I. Diskant, A generalization of Bonnesen's inequalities (Russian), Dokl. Akad. Nauk SSSR, 213 (1973), 519-521.

[5] J. R. SANGWINE-YANGER, Bonesen-style inequalities for Minkowski relative geometry, Trans. Amer. Math. Soc., 307 (1988), 373-382.

[6] L. A. Santaló, Integral Geometry and Geometry Probability, With a foreword by Mark Kac. Encyclopedia of Mathematics and its Applications, Vol. 1, Addison-Wesley Publishing Co., Reading, Mass.-London-Amsterdam, 1976.

[7] R. SchneIder, Convex bodies: the Brunn-Minkowski theory, Encyclopedia of Mathematics and its Applications 44, Cambridge University Press, Cambridge, 1993.

[8] R. Ossermann, Bonnesen-style Isoperimetric Inequalities, Amer. Math. Monthly, 86 (1979), 1-29.

[9] J. M. WiLls, Zum Verhältnis von Volume zu Oberfl äche bei konvexen körpen (German), Arch. Math. (Basel), 21 (1970), 557-560.

[10] J. ZHOU AND F. Chen, The Bonnesen-style inequality in a plane of constant curvature, J. Korean Math. Soc., 44 (2007), 1363-1372. 